\title{
Monoclonal Gammopathy of Undetermined Significance and Multiple Myeloma in Older Adults
}

Emily J. Guerard, $M D^{a}$, Sascha A. Tuchman, $M D^{b, *}$

\section{KEYWORDS}

$\bullet$ MGUS • Multiple myeloma • Older adult • Elderly • Geriatric • Cancer • Neoplasm

\section{KEY POINTS}

- Monoclonal gammopathy of undetermined significance and multiple myeloma (MM) are plasma cell disorders of advanced age.

- Unexplained anemia, hypercalcemia, renal insufficiency, or bone pain, among other symptoms, should prompt a work-up for a plasma cell disorder.

- Chemotherapy is standard of care for MM and, although incurable, survival in MM is progressively improving thanks to new drugs.

- Treating MM in older adults requires careful monitoring, dosing, and management of toxicity to attain good outcomes.

- Geriatric assessment and other novel instruments may soon enable personalization of MM therapy for older adults.

\section{INTRODUCTION AND EPIDEMIOLOGY}

Monoclonal gammopathy of undetermined significance (MGUS) and multiple myeloma (MM) are disorders on the spectrum of plasma cell dyscrasias that are diseases of aging. In multiple population-based studies, the prevalence of MGUS increases with age; $5.3 \%$ of people greater than or equal to 70 years old and $7.5 \%$ of people greater than or equal to 85 years old. ${ }^{1}$ The median age at diagnosis of MM

Disclosures: Dr E.J. Guerard has no disclosures. Dr S.A. Tuchman has performed consulting for Celgene and Takeda, and participated in speakers' bureau for Celgene and Takeda.

a Division of Hematology \& Oncology, Department of Medicine, University of North Carolina at Chapel Hill, 170 Manning Drive, Campus Box 7305, Chapel Hill, NC 27599, USA; b Division of Cellular Therapy and Hematologic Malignancies, Duke Cancer Institute, DUMC 3961, Durham, NC 27710, USA

* Corresponding author.

E-mail address: sat6@duke.edu 
is 69 years, with half of deaths from MM occurring in patients aged 75 years and older (Fig. 1). ${ }^{2}$ MM accounts for about $10 \%$ of all hematologic malignancies and $1.6 \%$ of all new cancer cases in the United States. ${ }^{2,3}$ It is estimated that there will be 26,850 new cases of MM in 2015. MGUS and MM are more common in men than in women, and in African Americans than in white people. ${ }^{1,2,4,5}$

Five-year overall survival in MM has increased from $29.7 \%$ in 1990 to $45.1 \%$ in $2007,{ }^{2}$ largely attributable to novel therapies. ${ }^{6}$

Given the anticipated growth in the older adult population and presumably static prevalence rates as a function of age, the raw numbers of older adults with plasma cell disorders will increase over time. Therefore, a familiarity with MM and MGUS is, and will remain, important for geriatricians.

\section{BIOLOGY AND CAUSE}

$\mathrm{MM}$ is a malignancy of plasma cells, terminally differentiated B lymphocytes that secrete antibodies when exposed to specific antigens. The exact pathogenesis of MM and MGUS is not well understood. Virtually all cases of MM are thought to arise from MGUS, a premalignant, asymptomatic proliferation of plasma cells. ${ }^{7,8}$ The initiation of MGUS is likely an abnormal plasma cell response to antigenic stimulation that leads to primary cytogenetic abnormalities and other genomic changes; the resultant derangement of plasma cell biology ultimately causes plasma cell clonal expansion and in some cases clinically significant disease. The expanded plasma cells usually overproduce a complete monoclonal immunoglobulin or some part thereof (eg, light chain only). 9,10 The transition in some cases from MGUS to MM is thought to be caused by additional biological abnormalities (eg, genomic, bone marrow microenvironmental) that lead to further clonal proliferation. ${ }^{9}$ Once MGUS has progressed to $\mathrm{MM}$, end organ damage begins because of infiltration of the neoplastic plasma cells into the bones and organ systems, damage mediated by circulating monoclonal

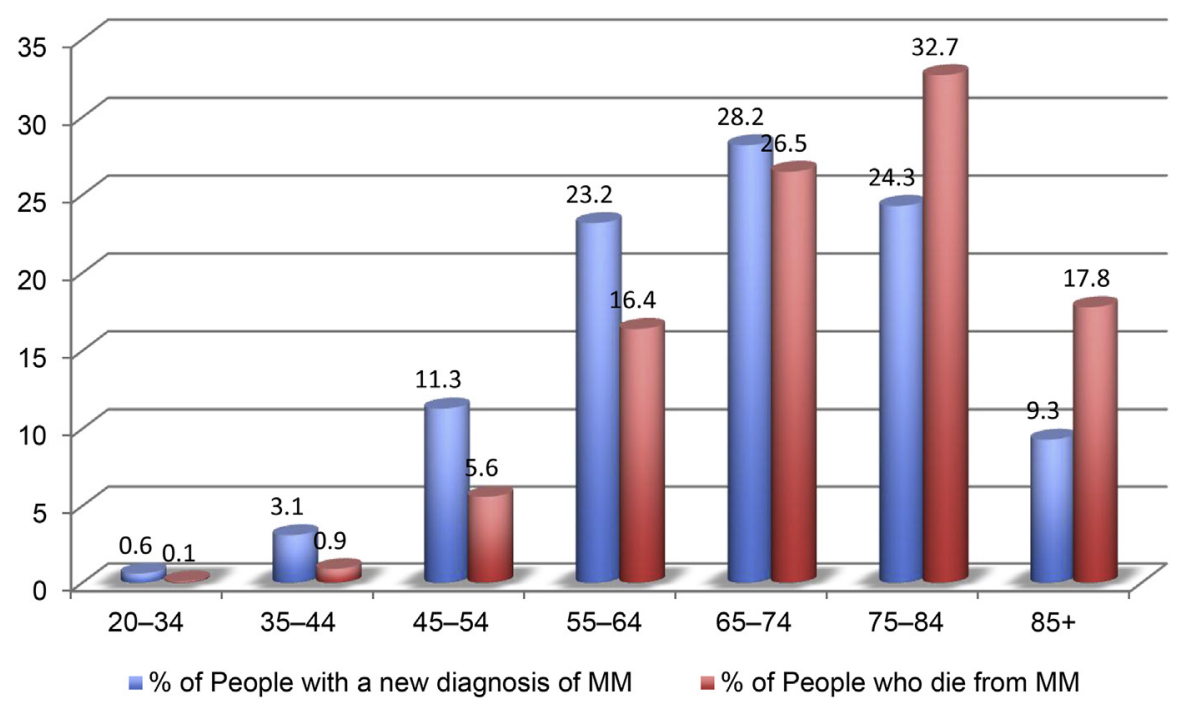

Fig. 1. Age distribution of patients with MM and deaths from MM. (Data from Institute NC. SEER stat fact sheets: myeloma. 2015. Available at: http://seer.cancer.gov/statfacts/html/ mulmy.html. Accessed May 18, 2015.) 
proteins (eg, cast nephropathy), or both. Between MGUS and MM there is an intermediate phase, termed smoldering MM (SMM), which is not always identified before an MM diagnosis. SMM constitutes a higher-burden disease state than MGUS, but one in which end organ damage is similarly absent.

Risk factors such as radiation or pesticide exposure, ${ }^{11,12}$ or history of autoimmune or chronic infectious disease,${ }^{13}$ have been described as risk factors for MM or MGUS, but most patients with MM and MGUS have no identifiable risk factors. Perhaps the most important risk factor for these diseases is advanced age. A growing body of research supports the link between aging and $\mathrm{MM}$; as an example, serum levels of the proinflammatory cytokine interleukin 6 (IL-6) increase as a function of age, ${ }^{14}$ and, in MM, plasma cells secrete IL-6 and overexpress the IL- 6 receptor. ${ }^{15}$ In the future, insights such as this are likely to elucidate deeper links between MM and aging and potentially yield beneficial interventions for both conditions.

\section{CLINICAL PRESENTATION OF MULTIPLE MYELOMA}

The clinical presentation of patients with MM is variable and can be subtle. The 2 case presentations discussed here highlight this variability.

- Case 1. A 75-year-old woman with osteoporosis presented to the emergency department after experiencing a fall followed by right hip pain. She reported fatigue, poor appetite, and mild weight loss for the past 6 months. Radiographs showed a pathologic fracture involving the distal femur. Intraoperative biopsy showed clonal plasma cells, and complete skeletal radiographs showed diffuse lytic bone lesions. Bone marrow biopsy also revealed clonal plasma cells, indicating a diagnosis of MM.

- Case 2. An 80-year-old man with hypertension and mild cognitive impairment presented to his geriatrician's office for routine follow-up. His only complaint was increased fatigue. Physical examination was nondiagnostic. Routine laboratory evaluation revealed normocytic anemia (hemoglobin level, $9.5 \mathrm{~g} / \mathrm{dL}$ ), increased total protein level, and a mild increase in his creatinine level to $1.35 \mathrm{mg} / \mathrm{dL}$. Serum protein electrophoresis (SPEP) and immunofixation revealed a monoclonal spike of $2.5 \mathrm{~g} / \mathrm{dL}$ immunoglobulin (lg) G-kappa, and a bone marrow aspirate and biopsy by a consulting hematologist showed $20 \%$ monoclonal plasma cells, confirming MM.

The common findings seen in MM at diagnosis are shown in Table $1 .{ }^{16}$ The lack of any clinical features that are frequently and uniquely present in $\mathrm{MM}$ can make this diagnosis challenging. For example, anemia is present in $73 \%$ of patients with $\mathrm{MM}$ but anemia is common in community-dwelling older adults and is more commonly explained by nutritional deficiencies, anemia of chronic disease, or chronic kidney disease. ${ }^{17}$ Hypercalcemia is present in $28 \%$ of patients with a new diagnosis of MM but is also commonly caused by thiazide diuretics or primary hyperparathyroidism. Fatigue, which is common in $\mathrm{MM}$, is seen in innumerable other medical conditions too. No physical examination findings are reliably sensitive and specific for diagnosing MM. ${ }^{16}$

\section{EVALUATION AND DIAGNOSIS}

Screening for MGUS and MM is not recommended for older adults. ${ }^{18}$ MGUS is highly prevalent, progresses to $\mathrm{MM}$ in only a small percentage of patients, ${ }^{19}$ and there is no known strategy to prevent that progression. ${ }^{18}$ Geriatricians should consider a diagnostic work-up for plasma cell disorders only when a reason to suspect one exists. 


\begin{tabular}{|ll}
\hline Table 1 & \\
Clinical features of MM & Frequency (\%) \\
Clinical Feature & \\
\hline History & 58 \\
\hline Bone pain & 42 \\
\hline Family history of cancer & 32 \\
\hline Fatigue & 24 \\
\hline Weight loss & 20 \\
\hline Known history of MGUS & 4 \\
\hline Physical Examination & 1 \\
\hline Hepatomegaly & 1 \\
\hline Splenomegaly & 73 \\
\hline Lymphadenopathy & 48 \\
\hline Laboratory & 28 \\
\hline Anemia & 20 \\
\hline Increased creatinine level & 5 \\
\hline Increased calcium level & \\
\hline Leukopenia & 66 \\
\hline Thrombocytopenia & 26 \\
\hline Radiology & 23 \\
\hline Lytic lesions & \\
\hline Pathologic fractures & \\
\hline Osteoporosis & \\
\hline
\end{tabular}

Data from Kyle RA, Gertz MA, Witzig TE, et al. Review of 1027 patients with newly diagnosed multiple myeloma. Mayo Clin Proc 2003;78(1):21-33.

The following list represents a sample of clinical findings that could trigger a diagnostic work-up:

- Age-disproportionate osteoporosis or osteopenia

- Increased serum total protein level without increased serum albumin level

- Bone pain, lytic bone lesions, pathologic and/or compression fractures

- Unexplained peripheral neuropathy, hypercalcemia, anemia, proteinuria, or renal insufficiency

The initial diagnostic work-up should include the following laboratory tests:

- SPEP and 24-hour urine protein electrophoresis (UPEP) with immunofixation

- Serum free light chains

- Complete blood count (CBC)

- Serum creatinine and calcium levels

- Quantitative immunoglobulins

The diagnostic criteria for MM, SMM, and MGUS were recently updated (Box 1). ${ }^{20}$ If a monoclonal protein is not detected on serum or urine studies, then MM is extremely unlikely and most plasma cell diseases can usually be confidently excluded from consideration; nonsecretory myeloma (ie, MM that does not secrete monoclonal protein) does exist but is rare. ${ }^{21}$ If a monoclonal protein is detected, then the patient needs additional work-up to clarify the diagnosis. The first step is to determine 
Box 1

2014 Revision of International Myeloma Working Group diagnostic criteria

$M M$

Clonal bone marrow plasma cells greater than or equal to $10 \%$, or biopsy-proven bone, or extramedullary plasmacytoma

Plus at least 1 myeloma-defining event.

Myeloma-defining events:

- Hypercalcemia

- Serum calcium level greater than $1 \mathrm{mg} / \mathrm{dL}$ higher than upper limit of normal or greater than $11 \mathrm{mg} / \mathrm{dL}$

- Renal insufficiency

- Creatinine clearance less than $40 \mathrm{~mL} / \mathrm{min}$ or serum creatinine level greater than $2 \mathrm{mg} / \mathrm{dL}$

- Anemia

- Hemoglobin level less than $10 \mathrm{~g} / \mathrm{dL}$ or greater than $2 \mathrm{~g} / \mathrm{dL}$ less than the lower limit of normal

- Bone lesions

- One or more osteolytic lesions on skeletal survey, computed tomography (CT), or PET/CT

- Biomarkers of malignancy

- Clonal bone marrow plasma cells greater than or equal to $60 \%$

- Involved/uninvolved serum free light chain ratio greater than 100 with involved light chain greater than $10 \mathrm{~g} / \mathrm{dL}$

- Greater than 1 focal lesion on MRI

\section{Smoldering $M M$}

Both criteria must be present:

- Serum monoclonal protein (IgG or IgA) level greater than $3 \mathrm{~g} / \mathrm{dL}$, or urinary monoclonal protein level greater than $500 \mathrm{mg}$ per 24 hours, and/or clonal bone marrow plasma cells $10 \%$ to $60 \%$

- No myeloma-defining events or evidence of amyloidosis

MGUS

All 3 criteria must be present:

- Serum monoclonal protein level less than $3 \mathrm{~g} / \mathrm{dL}$

- Bone marrow plasma cells less than $10 \%$

- No myeloma-defining events or evidence of amyloidosis

Adapted from Rajkumar SV, Dimopoulos MA, Palumbo A, et al. International Myeloma Working Group updated criteria for the diagnosis of multiple myeloma. Lancet Oncol 2014;15(12):e538-48.

whether the patient has at least 1 myeloma-defining event: hypercalcemia, renal insufficiency, anemia, bone lesions, or biomarkers of malignancy (see Box 1). Next, bone marrow aspiration and biopsy are indicated in patients with a myelomadefining event, a monoclonal protein level greater than $1.5 \mathrm{~g} / \mathrm{dL}$, a non-lgG monoclonal protein level of any amount, or abnormal free light chain ratio. In certain patients, further studies, including complete skeletal radiographs, lactate dehydrogenase, albumin, and $\beta 2$-microglobulin, should be considered. More sensitive imaging, such as computed tomography (CT) or MRI, should be used as warranted by clinical symptoms (eg, unexplained bone pain) to evaluate for lesions not detected by skeletal radiographs. 
If a patient is diagnosed with MGUS, the rate of progression to a true plasma cell cancer, usually $\mathrm{MM}$, is approximately $1 \%$ per year. ${ }^{19}$ There is no single prognostic marker to determine which patients will progress, but a serum monoclonal protein level of greater than $1.5 \mathrm{~g} / \mathrm{dL}$, a non-lgG monoclonal immunoglobulin, and abnormal serum free light chain ratio are known risk factors for progression (Table 2). ${ }^{22,23}$ Because the risk of progression does not decrease over time, patients with MGUS require lifelong monitoring. It has been suggested that patients with low-risk disease can be monitored by history and physical (HP) only for signs or symptoms of disease progression and do not necessarily need routine laboratory monitoring. ${ }^{24}$ All other risk groups should be monitored with an HP and laboratory evaluation with an SPEP, UPEP, CBC, creatinine level, and calcium level every 3 to 12 months, depending on the individual and the characteristics of the plasma cell disease. Older patients with a limited life expectancy ( $<5$ years) do not need routine follow-up given that these patients are likely to die of other causes before MGUS progresses to MM. Older adults with MGUS can be effectively monitored by their primary care physicians.

Unlike in MGUS, approximately half of patients with SMM progress to MM within 5 years and roughly $80 \%$ progress over 20 years. ${ }^{25}$ Risk factors for progression include an abnormal serum free light chain ratio or both a serum monoclonal protein level of greater than $3 \mathrm{~g} / \mathrm{dL}$ and clonal bone marrow plasma cells of greater than $10 \%$ (only 1 is required to diagnose SMM). ${ }^{25}$ Patients with SMM should be monitored at 2 to 3 months after the diagnosis and then every 4 to 6 months with an HP and laboratory testing. ${ }^{25}$ There are several therapies currently under investigation to prevent or delay progression to symptomatic $\mathrm{MM},{ }^{26}$ but standard of care in the United States arguably remains observation. Medical oncologists and hematologists are often involved in monitoring these higher-risk patients.

\section{STAGING AND PROGNOSIS}

The Durie-Salmon system was developed 40 years ago $^{27}$ and incorporates multiple known independent markers of prognosis (Box 2). Durie-Salmon still retains prognostic capacity. In one comparative study of patients after transplantation for MM, patients who were Durie-Salmon stage 1 versus stage 3 had a median overall survival of 82 versus 50 months, respectively. ${ }^{28}$ More recently, the simpler International Staging System (ISS) was developed (see Box 2). ${ }^{29}$ In the same study as described earlier, patients at ISS stage 1 versus 3 had overall survival of 64 and 45 months respectively. Notably, no study has definitively shown either system to be superior to the other, although ISS is used more commonly because of its simplicity. No large trial has

\begin{tabular}{|ll|}
\hline \multicolumn{2}{|l|}{ Table $\mathbf{2}$} \\
Mayo Clinic risk stratification for progression of MGUS to MM \\
\hline Risk Stratification & Risk of Progression at $\mathbf{2 0}$ y (\%) \\
\hline High risk (3 risk factors) & 58 \\
\hline High to intermediate risk (2 risk factors) & 37 \\
\hline Low to intermediate risk (1 risk factor) & 21 \\
\hline Low risk (0 risk factors) & 5 \\
\hline
\end{tabular}

Risk factors: serum monoclonal (M) protein level greater than $1.5 \mathrm{~g} / \mathrm{dL}$, non-lgG M-protein, abnormal serum free light chain ratio.

Adapted from Rajkumar SV, Kyle RA, Therneau TM, et al. Serum free light chain ratio is an independent risk factor for progression in monoclonal gammopathy of undetermined significance. Blood 2005; 106(3):812-7. 
Box 2

Prognostic systems in MM

Durie-Salmon staging system ${ }^{27}$

Stage 1

All of:

Hemoglobin level greater than $10 \mathrm{~g} / \mathrm{dL}$

Normal serum calcium level

Radiograph skeletal survey normal or showing solitary bone plasmacytoma or osteoporosis

Serum paraprotein level less than $5 \mathrm{~g} / \mathrm{dL}$ if $\mathrm{IgG}$, less than $3 \mathrm{~g} / \mathrm{dL}$ if $\lg A$

Urinary light chain excretion less than $4 \mathrm{~g} / 24 \mathrm{~h}$

Stage 2

Not stage 1 or 3

Stage 3

Any of:

Hemoglobin level less than $8.5 \mathrm{~g} / \mathrm{dL}$

Calcium level greater than $12 \mathrm{mg} / \mathrm{dL}$

Skeletal survey with more than 2 lytic lesions

Serum paraprotein level greater than $7 \mathrm{~g} / \mathrm{dL}$ if $\mathrm{IgG}$, greater than $5 \mathrm{~g} / \mathrm{dL}$ if $\lg A$

Urinary light chain excretion greater than $12 \mathrm{~g} / 24 \mathrm{~h}$

Subclassification:

A: Serum creatinine level less than $2 \mathrm{mg} / \mathrm{dL}$

B: Serum creatinine level greater than or equal to $2 \mathrm{mg} / \mathrm{dL}$

International Staging System ${ }^{29}$

Stage 1

Serum $\beta 2$-microglobulin level less than $3.5 \mathrm{mg} / \mathrm{dL}$ and serum albumin level greater than or equal to $3.5 \mathrm{~g} / \mathrm{dL}$

Stage 2

Not stage 1 or 3

Stage 3

Serum $\beta 2$-microglobulin level greater than or equal to $5.5 \mathrm{~g} / \mathrm{dL}$

Adapted from Durie BG, Salmon SE. A clinical staging system for multiple myeloma. Correlation of measured myeloma cell mass with presenting clinical features, response to treatment, and survival. Cancer 1975;36(3):842-54; and Greipp PR, San Miguel J, Durie BG, et al. International staging system for multiple myeloma. J Clin Oncol 2005;23(15):3412-20.

specifically examined the utility of ISS specifically in older patients with MM, but $34 \%$ of patients in the original report describing ISS were more than 65 years old, supporting the ability to generalize ISS to older adults with MM. ${ }^{29}$ Molecular markers are also highly prognostic in MM. Some, such as deletions of chromosome 17p (p53 gene), are common in many forms of cancer, whereas others, such as gains of chromosome 1q (CKS1B gene), are unique to MM. An extensive consensus discussion of molecular prognostic markers in MM has been recently published. ${ }^{30}$

Age is a prognostic marker in MM, albeit a complex one. Multiple population-level studies show that survival in older patients with MM is inferior to that of younger patients, despite improvements over recent decades in all age groups. ${ }^{31-33}$ However, it is unclear to what degree poorer survival in older adults is caused by differences in 
disease biology, inability to tolerate intensive therapy such as transplant, medical comorbidity, or simply natural death caused by advanced age. MM biology does not seem to be distinctly different in younger versus older patients, in contrast with other hematological malignancies such as acute myelogenous leukemia, in which advanced age predicts high-risk biology. ${ }^{34}$ In MM, for example, a balanced translocation of chromosomes 4 and 14 , termed $t(4 ; 14)$, is a marker of poor prognosis that in one study was less common in older patients, ${ }^{35}$ but in another report ISS stages with good prognosis were also less common in older patients. ${ }^{36}$ Hence the scarce available data are contradictory and further studies are needed to elucidate the interaction between age and MM biology in more detail.

\section{MANAGEMENT AND TREATMENT}

Treating MM in older adults can be affected by several factors that have little to do with MM itself, such as medical comorbidity, polypharmacy, and frailty. This issue is critical given that the intensity of MM therapy ranges from low-dose, single drugs intended to achieve disease palliation and minimize toxicity, to high-dose chemotherapy with autologous hematopoietic stem cell transplant (ASCT). Treating clinicians need to carefully judge the capacity of each older adult to tolerate the different levels of intensity of possible MM therapies. Overly generous assessments of a patient's capacity to tolerate therapy may result in overtreatment and excessive, even fatal, toxicity. Overly conservative assessments could result in inadequately intensive therapy and increased risk of poor disease response, early relapse, and disease-related morbidity or mortality. Hence much of the focus in recent years has been on optimizing geriatricspecific assessment techniques for predicting therapeutic toxicity, with the overarching goal of matching individual older adults with regimens of an intensity that best controls MM without inducing serious harm.

The arguable gold standard for maximally intensive MM therapy is ASCT. Historically, age less than 65 years was the key determinant for ASCT candidacy, enforced largely by lack of governmental insurance coverage for ASCT for patients more than 65 years old in Europe. Such policy limitations did not exist in the United States, although strict age cutoffs were observed in most transplant centers.

More recently, physiologic age is increasingly recognized as superior to chronologic age for assessing ASCT candidates. Several studies have shown that ASCT with dose-reduced chemotherapy can be safe and efficacious in appropriately selected older adults (usually those with limited comorbidities and good functional status), with similar rates of severe toxicity, time until MM progression, and overall survival compared with younger patients. ${ }^{37-39}$ In particular, fit patients in their early 70 s are now routinely offered ASCT with a safe expectation of good results.

A comprehensive discussion of drug regimens for the treatment of $\mathrm{MM}$ goes beyond the scope of this article. However, a basic listing of some of the more common treatment regimens for older adults with $\mathrm{MM}$ is shown in Table 3. The primary drug classes include corticosteroids (dexamethasone or prednisone), conventional cytotoxic drugs (primarily the alkylators cyclophosphamide and melphalan), and newer drugs (ie, novel agents). The 2 classes of novel agents approved for commercial use are proteasome inhibitors (bortezomib and carfilzomib) and immunomodulatory agents (thalidomide, lenalidomide, and pomalidomide). Even newer drugs in those therapeutic classes are being developed, as are drugs from new classes, such as monoclonal antibodies. At the time of writing, daratumumab (targets CD38) and elotuzumab (targets SLAMF7) are examples of monoclonal antibodies currently under US Food and Drug Administration (FDA) review for possible approval for treatment of relapsed and refractory MM. 


\section{Table 3}

Common treatment regimens for older adults with MM

\begin{tabular}{|c|c|c|}
\hline \multicolumn{2}{|c|}{ Initial Treatment Options } & $\begin{array}{l}\text { Relapsed/Refractory } \\
\text { Drug Options }^{\text {a }}\end{array}$ \\
\hline $\begin{array}{l}\text { Transplant Eligible } \\
\text { - Bortezomib, lenalidomide, } \\
\text { dexamethasone } \\
\text { - Bortezomib, cyclophosphamide, } \\
\text { dexamethasone } \\
\text { - Bortezomib, dexamethasone } \\
\text { - Lenalidomide, dexamethasone }\end{array}$ & $\begin{array}{l}\text { Transplant Ineligible } \\
\text { - Any regimen recommended } \\
\text { for transplant eligible } \\
\text { - Bortezomib, melphalan, } \\
\text { prednisone } \\
\text { - Melphalan, prednisone, } \\
\text { thalidomide } \\
\text { - Melphalan, prednisone, } \\
\text { lenalidomide }\end{array}$ & $\begin{array}{l}\text { Immunomodulators } \\
\text { - Lenalidomide } \\
\text { - Pomalidomide } \\
\text { - Thalidomide } \\
\text { Proteasome inhibitors } \\
\text { - Bortezomib } \\
\text { - Carfilzomib } \\
\text { Alkylators } \\
\text { - Cyclophosphamide } \\
\text { - Melphalan } \\
\text { Other cytotoxics } \\
\text { - Doxorubicin (standard } \\
\text { - or liposomal) } \\
\text { - Vincristine } \\
\text { - Bendamustine } \\
\text { Corticosteroids } \\
\text { - Dexamethasone } \\
\text { - Prednisone }\end{array}$ \\
\hline
\end{tabular}

a A combination of drugs is commonly used in the relapsed/refractory setting and the choice of drug combinations is based on previous treatments' efficacy and tolerability, patient-related factors, and perceived disease aggressiveness, among many other factors.

The choice of the treatment regimen depends on whether the patient is thought to be a suitable candidate for ASCT, other patient-related factors, and MM-specific factors, such as the presence of specific genetic abnormalities. However, the lines between regimens specifically for ASCT candidates versus noncandidates are blurring. Historically, melphalan was a favorite among non-ASCT candidates but was avoided in ASCT candidates because of melphalan's hematopoietic stem cell toxicity and its propensity to impair stem cell mobilization. More recently, melphalan's usage has decreased as other highly effective drugs have become available, resulting in less firm distinction between regimens for these two cohorts of patients. Another important point is that most of these regimens were not studied extensively in much older adults and/or those with substantial comorbidities, so oncologists commonly reduce doses and use alternative administration strategies based their assessment of each older patient.

Further building on the concept that to some degree age is just a number, cancerspecific geriatric assessment (GA) instruments have been developed that evaluate older adults in a manner that uncovers problems not routinely identified by oncologists, predicts chemotherapy toxicity, and in the future may assist in therapy selection. The Cancer and Aging Research Group (CARG) GA was developed for older patients with cancer and contains valid and reliable measures of geriatric domains pertaining to physical function, independent activities of daily living (IADLs), polypharmacy, medical comorbidity, nutritional status, mental health, social support, and cognitive function. ${ }^{40}$ The CARG GA, which is mostly patient reported, was shown to predict chemotherapy toxicity. High-risk versus low-risk patients identified by the CARG toxicity tool had an $89 \%$ versus $25 \%$ risk respectively of severe chemotherapy toxicity, in contrast with provider-reported Karnofsky Performance Status, which did not predict toxicity. ${ }^{41}$ Another model, the Chemotherapy Risk Assessment Scale for High-Age Patients 
(CRASH), has also been developed as a toxicity predictor and incorporates both chemotherapy regimen-specific risk of toxicity and patient-specific predictors, such as performance status, mini-nutritional assessment, and mini-mental health status. ${ }^{42}$ Clearly GA tools have great promise in MM, but an important caveat is that these GA instruments have generally been tested in patients with solid tumors and their relevance to MM has not been fully elucidated. Recently, a single larger study of older adults with MM used a combination of age, the Charlson Comorbidity Index, and ability to perform IADLs to classify patients as frail, intermediate fitness, and fit. That model predicted vital MM-specific outcomes: toxicity, discontinuation of therapy within 12 months, progression-free survival, and overall survival (Table 4). ${ }^{43}$ This study serves as proof of principle of GA's relevance to MM, but presumably more comprehensive GA instruments such as CARG or CRASH could even better classify at-risk patients.

Observational studies are ongoing to test more comprehensive GA systems such as the CARG GA directly in MM, and initial data are promising. ${ }^{44}$ The next challenge is to incorporate GA-derived predictions of chemotherapy toxicity into decision-making models for therapy selection in older adults with MM. If so, GA may someday provide an avenue to match older MM patients with treatment regimens of appropriate intensity, thereby maximizing the likelihood of $\mathrm{MM}$ control and avoiding overtreating or undertreating and risking severe toxicity or inadequate efficacy respectively. Only now are groups such as ours and others designing clinical trials that incorporate some form of GA into eligibility criteria. Trials such as these that use GA to determine treatment intensity may prepare the way to GA-based decision models for therapy selection and allow prospective assessments of the effect a drug has on GA domains, which are important and unique outcomes to older patients with cancer.

\section{SIDE EFFECTS AND SUPPORTIVE CARE}

Older adults are subject to the same toxicities that younger patients may experience while undergoing therapy for $\mathrm{MM}$, and, for the reasons discussed earlier, such as reduced homeostatic reserve and medical comorbidities, therapy-related toxicities can be more pronounced in older patients. Therefore, aggressive supportive care and a deep familiarity with the side effect profile of MM therapies and management strategies are critical to successful therapy. Certain unique issues that stem from widely used drugs in MM warrant mentioning when considering treatment of older adults.

Bortezomib (Velcade) is broadly used and FDA approved in both newly diagnosed and relapsed $\mathrm{MM}$, with data from large randomized trials showing prolonged remission and overall survival. ${ }^{45,46}$ Common toxicities include sometimes severe gastrointestinal

\begin{tabular}{|c|c|c|c|c|}
\hline \multicolumn{5}{|c|}{$\begin{array}{l}\text { Table } 4 \\
\text { International Myeloma Working Group GA and outcomes }\end{array}$} \\
\hline GA Classification & $\begin{array}{l}\text { Overall Survival (Hazard } \\
\text { Ratio }^{\text {a) }}\end{array}$ & $P$ & $\begin{array}{l}\text { Therapy Discontinuation } \\
\text { Within } 12 \mathrm{mo}(\%)\end{array}$ & $P$ \\
\hline Fit & 1 & - & 1 & - \\
\hline Intermediate fitness & 1.37 & .181 & 1.41 & .52 \\
\hline Frail & 2.88 & $<.001$ & 2.21 & $<.001$ \\
\hline
\end{tabular}

a Adjusted for therapy, chromosomal abnormalities, and ISS stage.

Data from Palumbo A, Bringhen S, Mateos MV, et al. Geriatric assessment predicts survival and toxicities in elderly myeloma patients: an International Myeloma Working Group report. Blood 2015;125(13):2068-74. 
effects (usually diarrhea and less often constipation), sensory peripheral neuropathy, and orthostatic hypotension. Studies have shown that administering bortezomib once weekly instead of twice weekly reduces gastrointestinal and neuropathic toxicity by roughly $30 \%,{ }^{47}$ and subcutaneous instead of intravenous administration similarly reduces toxicity. ${ }^{48}$ Neither modification detracts from bortezomib's overall treatment efficacy, and decisions about dosing are usually determined by the patient's health status and disease characteristics. Providers and nurses need to routinely assess side effects and aggressively modify doses if such toxicity appears to a substantial degree. Bortezomib-induced neuropathy can be reversible with dose modifications or cessation. ${ }^{49}$ Antimotility agents such as loperamide can control diarrhea. Patients who regularly experience diarrhea after bortezomib can use antimotility agents preemptively before diarrhea starts. In addition, paying close attention to hydration/volume status and electrolytes is vital.

In the past, thalidomide was often used to treat MM and detailed discussions of toxicity were required. Thalidomide commonly causes constipation, sedation, and peripheral neuropathy, which can be particularly problematic in older adults. Readily available alternatives in the same drug class of immunomodulatory agents, lenalidomide (Revlimid) and pomalidomide (Pomalyst), are generally more efficacious and less toxic than thalidomide. ${ }^{50-53}$ Therefore, the best thalidomide-based strategy for seniors with $\mathrm{MM}$ is probably to consider avoiding the drug entirely by selecting a different agent from the same class. In the uncommon circumstance that thalidomide is required, caution is warranted. The toxicities of lenalidomide and pomalidomide are primarily cytopenias, fatigue, and in some cases rash. ${ }^{50-53}$

High-dose corticosteroids, usually dexamethasone (Decadron) or prednisone, are ubiquitous in MM regimens and for some patients are the most intolerable component of therapy. The adoption of low-dose dexamethasone (usually once weekly) as the standard for most regimens instead of the prior standard of high-dose dexamethasone (usually 4 days on, 4 days off) has mitigated steroid toxicity in $\mathrm{MM}^{54}$ but delirium, insomnia, anxiety, weight gain caused by polyphagia, hyperglycemia, peripheral edema, and peptic ulcers are still common. These toxicities can sometimes be controlled through supportive care, but often remain difficult and dose reduction is the safest course of action. Peptic ulcer disease prophylaxis is useful, because many patients are on both dexamethasone and aspirin as part of their MM regimens and both drugs predispose to ulcers and/or upper gastrointestinal bleeding.

Expert opinions regarding recommended starting doses for various drugs in MM have been offered by our group as well as the European Myeloma Network in recent years. ${ }^{55,56}$ More direct evidence is needed to further optimize treatment of older adults with MM.

As for supportive care, older adults should also receive the same interventions as younger patients with MM: herpes zoster prophylaxis with proteasome inhibitors and thromboembolic prophylaxis with aspirin, or anticoagulation if additional risk factors for venous thromboembolism are present. Most patients with MM should receive intravenous bisphosphonates given their capacity to reduce fractures and also prolong overall survival in $\mathrm{MM}{ }^{57}$ Before initiation of bisphosphonates, patients should be evaluated by a dentist given the association between osteonecrosis of the jaw (ONJ) and poor dentition. Major dental work (eg, extractions and implants) should be avoided if possible in patients receiving intensive bisphosphonate therapy. Periprocedural antibiotic prophylaxis has also been shown to reduce the risk of $\mathrm{ONJ}$ in observational studies. A recent set of guidelines covers this topic at length. ${ }^{58}$ Most patients with MM but not hypercalcemia should take calcium and vitamin D supplementation for bone health. ${ }^{59}$ 
Support through cytopenias is similar to that given to younger patients with MM and similar strategies using chemotherapy dose reductions/omissions, growth factors, and/or transfusions can be used. Special attention should be paid to the combined effects of anemia, hypovolemia, and/or autonomic dysfunction to avoid orthostatic hypotension, which can result in syncope or falls.

Because many older adults with MM die from their disease, palliative and hospice care services are used frequently. Early palliative care involvement for patients with challenging symptoms is important. Caregiver burden can be substantial in MM because many patients are on complex treatment regimens, require frequent clinic visits, and have difficulties coping with the illness. ${ }^{60}$ Providers need to be aware of these issues and provide appropriate supportive services when needed and available.

\section{SUMMARY}

MM and MGUS are diseases of the elderly and frequently appear in the geriatrician's clinic. A keen awareness of signs or symptoms suggesting the presence of a plasma cell disorder may prompt discovery of MM before the development of incapacitating sequelae, such as pathologic fractures. Standard of care for MGUS and SMM is monitoring, whereas chemotherapy is standard for MM. Novel therapeutics and ASCT have improved survival in MM but special caution is warranted when treating older adults with $\mathrm{MM}$ because of the unique toxicity profiles of MM drugs, which can combine with preexisting medical issues to produce adverse outcomes. Novel assessment tools such as GA may facilitate the personalization of older patients' MM treatment programs in the future, maximizing the likelihood of MM control and minimizing the risk of severe toxicity. Geriatricians are uniquely positioned to detect these diseases early, support patients through treatment, and aid oncologists with an assessment of the patient's physiologic/functional age to provide optimal care for older adults with these diseases.

\section{REFERENCES}

1. Kyle RA, Therneau TM, Rajkumar SV, et al. Prevalence of monoclonal gammopathy of undetermined significance. N Engl J Med 2006;354(13):1362-9.

2. Institute NC. SEER stat fact sheets: myeloma. 2015. Available at: http://seer. cancer.gov/statfacts/html/mulmy.html. Accessed May 18, 2015.

3. Siegel RL, Miller KD, Jemal A. Cancer statistics, 2015. CA Cancer J Clin 2015; 65(1):5-29.

4. Waxman AJ, Mink PJ, Devesa SS, et al. Racial disparities in incidence and outcome in multiple myeloma: a population-based study. Blood 2010;116(25): $5501-6$.

5. Landgren O, Gridley G, Turesson I, et al. Risk of monoclonal gammopathy of undetermined significance (MGUS) and subsequent multiple myeloma among African American and white veterans in the United States. Blood 2006;107(3):904-6.

6. Kumar SK, Rajkumar SV, Dispenzieri A, et al. Improved survival in multiple myeloma and the impact of novel therapies. Blood 2008;111(5):2516-20.

7. Weiss BM, Abadie J, Verma P, et al. A monoclonal gammopathy precedes multiple myeloma in most patients. Blood 2009;113(22):5418-22.

8. Landgren O, Kyle RA, Pfeiffer RM, et al. Monoclonal gammopathy of undetermined significance (MGUS) consistently precedes multiple myeloma: a prospective study. Blood 2009;113(22):5412-7.

9. Rajkumar SV. Prevention of progression in monoclonal gammopathy of undetermined significance. Clin Cancer Res 2009;15(18):5606-8. 
10. Fonseca R, Bergsagel PL, Drach J, et al. International Myeloma Working Group molecular classification of multiple myeloma: spotlight review. Leukemia 2009; 23(12):2210-21.

11. Iwanaga M, Tagawa M, Tsukasaki K, et al. Relationship between monoclonal gammopathy of undetermined significance and radiation exposure in Nagasaki atomic bomb survivors. Blood 2009;113(8):1639-50.

12. Cantor KP, Blair A. Farming and mortality from multiple myeloma: a case-control study with the use of death certificates. J Natl Cancer Inst 1984;72(2):251-5.

13. van de Donk NW, Palumbo A, Johnsen HE, et al. The clinical relevance and management of monoclonal gammopathy of undetermined significance and related disorders: recommendations from the European Myeloma Network. Haematologica 2014;99(6):984-96.

14. Ershler WB. Interleukin-6: a cytokine for gerontologists. J Am Geriatr Soc 1993; 41(2):176-81.

15. Rawstron AC, Fenton JA, Ashcroft J, et al. The interleukin-6 receptor alpha-chain (CD126) is expressed by neoplastic but not normal plasma cells. Blood 2000; 96(12):3880-6.

16. Kyle RA, Gertz MA, Witzig TE, et al. Review of 1027 patients with newly diagnosed multiple myeloma. Mayo Clin Proc 2003;78(1):21-33.

17. Guralnik JM, Eisenstaedt RS, Ferrucci L, et al. Prevalence of anemia in persons 65 years and older in the United States: evidence for a high rate of unexplained anemia. Blood 2004;104(8):2263-8.

18. Berenson JR, Anderson KC, Audell RA, et al. Monoclonal gammopathy of undetermined significance: a consensus statement. Br J Haematol 2010;150(1):28-38.

19. Kyle RA, Therneau TM, Rajkumar SV, et al. A long-term study of prognosis in monoclonal gammopathy of undetermined significance. N Engl J Med 2002;346(8):564-9.

20. Rajkumar SV, Dimopoulos MA, Palumbo A, et al. International Myeloma Working Group updated criteria for the diagnosis of multiple myeloma. Lancet Oncol 2014;15(12):e538-48.

21. Dispenzieri A, Kyle R, Merlini G, et al. International Myeloma Working Group guidelines for serum-free light chain analysis in multiple myeloma and related disorders. Leukemia 2009;23(2):215-24.

22. Rajkumar SV, Kyle RA, Therneau TM, et al. Serum free light chain ratio is an independent risk factor for progression in monoclonal gammopathy of undetermined significance. Blood 2005;106(3):812-7.

23. Cesana C, Klersy C, Barbarano L, et al. Prognostic factors for malignant transformation in monoclonal gammopathy of undetermined significance and smoldering multiple myeloma. J Clin Oncol 2002;20(6):1625-34.

24. Bianchi G, Kyle RA, Colby CL, et al. Impact of optimal follow-up of monoclonal gammopathy of undetermined significance on early diagnosis and prevention of myeloma-related complications. Blood 2010;116(12):2019-25 [quiz: 2197].

25. Kyle RA, Remstein ED, Therneau TM, et al. Clinical course and prognosis of smoldering (asymptomatic) multiple myeloma. N Engl J Med 2007;356(25):2582-90.

26. Mateos MV, Hernandez MT, Giraldo P, et al. Lenalidomide plus dexamethasone for high-risk smoldering multiple myeloma. N Engl J Med 2013;369(5):438-47.

27. Durie BG, Salmon SE. A clinical staging system for multiple myeloma. Correlation of measured myeloma cell mass with presenting clinical features, response to treatment, and survival. Cancer 1975;36(3):842-54.

28. Hari PN, Zhang M-J, Roy V, et al. Is the International Staging System superior to the Durie-Salmon staging system? A comparison in multiple myeloma patients undergoing autologous transplant. Leukemia 2009;23(8):1528-34. 
29. Greipp PR, San Miguel J, Durie BG, et al. International staging system for multiple myeloma. J Clin Oncol 2005;23(15):3412-20.

30. Chng WJ, Dispenzieri A, Chim C-S, et al. IMWG consensus on risk stratification in multiple myeloma. Leukemia 2014;28(2):269-77.

31. Kumar SK, Dispenzieri A, Lacy MQ, et al. Continued improvement in survival in multiple myeloma: changes in early mortality and outcomes in older patients. Leukemia 2013;28(5):1122-8.

32. Pulte D, Gondos A, Brenner H. Improvement in survival of older adults with multiple myeloma: results of an updated period analysis of SEER data. Oncologist 2011;16(11):1600-3.

33. Brenner H, Gondos A, Pulte D. Recent major improvement in long-term survival of younger patients with multiple myeloma. Blood 2008;111(5):2521-6.

34. Wahlin A, Markevärn B, Golovleva I, et al. Prognostic significance of risk group stratification in elderly patients with acute myeloid leukaemia. Br J Haematol 2001;115(1):25-33.

35. Avet-Loiseau H, Hulin C, Campion L, et al. Chromosomal abnormalities are major prognostic factors in elderly patients with multiple myeloma: the Intergroupe Francophone du Myélome experience. J Clin Oncol 2013;31(22):2806-9.

36. Ludwig H, Durie BGM, Bolejack V, et al. Myeloma in patients younger than age 50 years presents with more favorable features and shows better survival: an analysis of 10549 patients from the International Myeloma Working Group. Blood 2008;111(8):4039-47.

37. Kumar SK, Dingli D, Lacy MQ, et al. Autologous stem cell transplantation in patients of 70 years and older with multiple myeloma: results from a matched pair analysis. Am J Hematol 2008;83(8):614-7.

38. Miller CB, Piantadosi S, Vogelsang GB, et al. Impact of age on outcome of patients with cancer undergoing autologous bone marrow transplant. J Clin Oncol 1996;14(4): 1327-32.

39. Bashir Q, Shah N, Parmar S, et al. Feasibility of autologous hematopoietic stem cell transplant in patients aged $\geq 70$ years with multiple myeloma. Leuk Lymphoma 2012;53(1):118-22.

40. Hurria A, Gupta S, Zauderer M, et al. Developing a cancer-specific geriatric assessment: a feasibility study. Cancer 2005;104(9):1998-2005.

41. Hurria A, Togawa K, Mohile SG, et al. Predicting chemotherapy toxicity in older adults with cancer: a prospective multicenter study. J Clin Oncol 2011;29(25): 3457-65.

42. Extermann M, Boler I, Reich RR, et al. Predicting the risk of chemotherapy toxicity in older patients: the Chemotherapy Risk Assessment Scale for High-Age Patients (CRASH) score. Cancer 2012;118(13):3377-86.

43. Palumbo A, Bringhen S, Mateos M-V, et al. Geriatric assessment predicts survival and toxicities in elderly myeloma patients: an International Myeloma Working Group report. Blood 2015;125(13):2068-74.

44. Wildes T, Tuchman SA. Geriatric assessment (GA) and eligibility for autologous stem cell transplant (ASCT) in older adults with newly diagnosed multiple myeloma (MM). Paper presented at: Journal of Clinical Oncology. Chicago, IL, 2015.

45. Mateos MV, Richardson PG, Schlag R, et al. Bortezomib plus melphalan and prednisone compared with melphalan and prednisone in previously untreated multiple myeloma: updated follow-up and impact of subsequent therapy in the phase III VISTA trial. J Clin Oncol 2010;28(13):2259-66.

46. Richardson PG, Barlogie B, Berenson J, et al. A phase 2 study of bortezomib in relapsed, refractory myeloma. N Engl J Med 2003;348(26):2609-17. 
47. Bringhen S, Larocca A, Rossi D, et al. Efficacy and safety of once-weekly bortezomib in multiple myeloma patients. Blood 2010;116(23):4745-53.

48. Moreau P, Pylypenko H, Grosicki S, et al. Subcutaneous versus intravenous administration of bortezomib in patients with relapsed multiple myeloma: a randomised, phase 3, non-inferiority study. Lancet Oncol 2011;12(5):431-40.

49. Richardson PG, Sonneveld P, Schuster MW, et al. Reversibility of symptomatic peripheral neuropathy with bortezomib in the phase III APEX trial in relapsed multiple myeloma: impact of a dose-modification guideline. Br J Haematol 2009; 144(6):895-903.

50. Gay F, Hayman SR, Lacy MQ, et al. Lenalidomide plus dexamethasone versus thalidomide plus dexamethasone in newly diagnosed multiple myeloma: a comparative analysis of 411 patients. Blood 2010;115(7):1343-50.

51. Weber DM, Chen C, Niesvizky R, et al. Lenalidomide plus dexamethasone for relapsed multiple myeloma in North America. N Engl J Med 2007;357(21): 2133-42.

52. Benboubker L, Dimopoulos MA, Dispenzieri A, et al. Lenalidomide and dexamethasone in transplant-ineligible patients with myeloma. N Engl J Med 2014; 371(10):906-17.

53. Leleu X, Attal M, Arnulf B, et al. Pomalidomide plus low-dose dexamethasone is active and well tolerated in bortezomib and lenalidomide-refractory multiple myeloma: Intergroupe Francophone du Myelome 2009-02. Blood 2013;121(11): 1968-75.

54. Rajkumar SV, Jacobus S, Callander NS, et al. Lenalidomide plus high-dose dexamethasone versus lenalidomide plus low-dose dexamethasone as initial therapy for newly diagnosed multiple myeloma: an open-label randomised controlled trial. Lancet Oncol 2010;11(1):29-37.

55. Wildes TM, Rosko A, Tuchman SA. Multiple myeloma in the older adult: better prospects, more challenges. J Clin Oncol 2014;32(24):2531-40.

56. Palumbo A, Bringhen S, Ludwig H, et al. Personalized therapy in multiple myeloma according to patient age and vulnerability: a report of the European Myeloma Network (EMN). Blood 2011;118(17):4519-29.

57. Morgan GJ, Child JA, Gregory WM, et al. Effects of zoledronic acid versus clodronic acid on skeletal morbidity in patients with newly diagnosed multiple myeloma (MRC Myeloma IX): secondary outcomes from a randomised controlled trial. Lancet Oncol 2011;12(8):743-52.

58. Khan AA, Morrison A, Hanley DA, et al. Diagnosis and management of osteonecrosis of the jaw: a systematic review and international consensus. J Bone Miner Res 2015;30(1):3-23.

59. Forrest KYZ, Stuhldreher WL. Prevalence and correlates of vitamin D deficiency in US adults. Nutr Res 2011;31(1):48-54.

60. Molassiotis A, Wilson B, Blair S, et al. Living with multiple myeloma: experiences of patients and their informal caregivers. Support Care Cancer 2011;19(1): 101-11. 